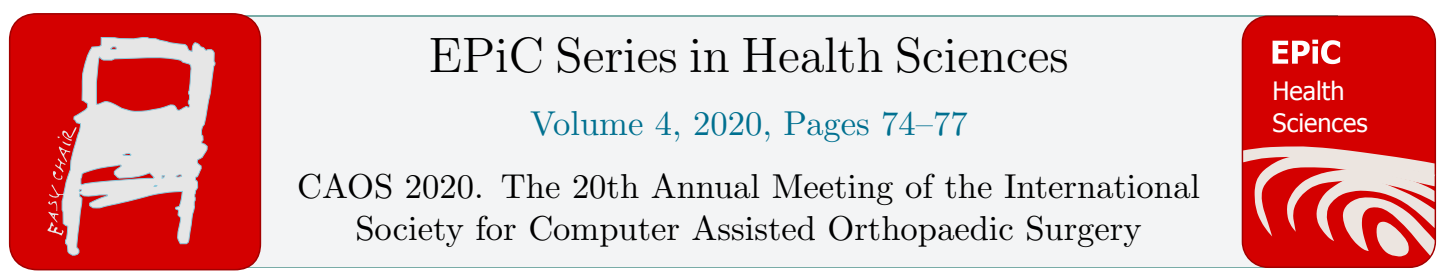

\title{
CAOS TKA Provides Improved Functional Outcomes Compared to Conventional TKA
}

\author{
Wen Fan ${ }^{1}$, Yifei Dai ${ }^{1}$, Gérard Giordano ${ }^{2 *}$ \\ ${ }^{1 *}$ Exactech Inc, Gainesville, FL 32653, USA \\ ${ }^{2}$ Hôpital Joseph Ducuing, Toulouse, FR \\ ortho.giordano@gmail.com
}

\begin{abstract}
This study examined the advantages of CAOS TKA in short-term functional outcomes compared to conventional instrumented TKA. Data were collected from a prospective, multi-center study where 334 were treated with CAOS TKA, and 461 were treated with conventional TKA. Postoperatively at 2-3 year, the CAOS cases had significantly better range of motion (ROM), KSS function, and sub-component measures of KSS function than the conventional cases. Further analysis revealed that geographic region was associated with ROM but had no significant impact on KSS function. CAOS TKA was associated with higher postoperative KSS function and its sub-component measures. The short-term results reported support the use of CAOS technology with the goals of better function.
\end{abstract}

\section{Introduction}

Computer-assisted orthopedic surgery (CAOS) has been shown to offer clear advantage regarding surgical accuracy in total knee arthroplasty (TKA), with a body of research studies demonstrating significant reduction of alignment outliers compared to conventional TKA [1-2]. However, conflicted data exists in the literature for a consensus regarding the advantage of CAOS technology in clinical outcomes or satisfaction rates for the patient. While some studies have shown superior functional outcomes in CAOS TKA compared to its conventional counterparts [3], others reported no difference between CAOS and conventional cases [4]. More studies are needed to further contribute knowledge and evidences to this topic. The objective of this study was to compare short-term clinical outcomes between TKA cases performed using a contemporary CAOS system and cases with conventional instrumentation. 


\section{Materials and Methods}

With institutional review board-approval and patient's signed informed consent, a prospective, multicenter, consecutive TKA case series was collected by 3 surgeons from 3 different clinical sites [2 US sites, $1 \mathrm{EU}$ site] using the same implant system. Seven hundred and ninety-five patients were enrolled with surgery date between November 2009 and September 2018, including 334 CAOS TKA cases and 461 conventional TKA cases. Each surgeon performed both CAOS and conventional surgeries. Patient demographics, baseline clinical measurements, and the latest minimum 1yr followup visit were reviewed and compared between the CAOS TKA group and the conventional TKA group. The clinical measurements investigated were Range of Motion (ROM), Knee Society Score (KSS: knee, function, pain, and each sub-component measure), and patient satisfaction Visual Analog Scale (VAS 1-10, with 10 indicating the highest satisfaction). All data analyses were performed using custom scripts in R 3.6.1 (RStudio, Inc., Boston, MA, USA). Two-sample t-test was used for continuous outcomes, and chi-squared test was used for binary outcomes. To further assess the detected postoperative significance, a multivariate regression analysis was performed to assess the impact of region (EU vs US) and treatment type (CAOS vs conventional). Statistical significance was defined as $\mathrm{p} \leq 0.05$.

\section{Results}

At the time of study, 215 CAOS and 350 conventional patients were available for analysis of patient reported outcomes, with mean postoperative follow-up periods of approximately 2-3 year (Table 1A). Patients from CAOS group were older and had higher BMI than those from conventional group ( $\mathrm{p}$ values < 0.01, Table 1A). Preoperatively in the baseline measures, although CAOS patients had higher KSS function score than patients in conventional group, no significant difference was found in each sub-component measure under KSS function ("Walking", "Stairs", and "Walking Aid") (Fig 1). No other differences existed between the two groups regarding patient characteristics and preoperative baseline.

Postoperatively, significant higher ROM was achieved in the CAOS group compared to the conventional group ( $\mathrm{p}<0.01$, Table 1B). In addition, higher KSS function score were found in CAOS compared to conventional group ( $\mathrm{p}<0.01$, Table 1B). The differences were further expressed in the sub-component measures. Compared to the conventional patients, CAOS patients scored significantly better in all sub-component measures ( $\mathrm{p}$ values $\leq 0.05$, Fig 2). No difference was found in KSS knee, and KSS pain scores. Although EU patients was found associated with higher postoperative ROM, geographic region was not significantly correlated with KSS function and its sub-component measures. In contrast, CAOS surgery was significantly associated with better KSS function and its sub-component measures ("Walking" and "Stairs") compared to conventional surgery ( $\mathrm{p}$ values $<0.04$ ). Both groups achieved a mean satisfaction rate of 9 (N.S.). Fourteen conventional cases were revised due to pain (5), loosening (3), infection (2), instability (1), and patellofemoral complications (3). Four knees in the CAOS group were revised due to infection (4).

\section{Discussion}

This study demonstrated significantly better short-term functional outcome for the patients received CAOS TKA compared to those received conventional TKA. Aligned with the reports from previous studies [3,5], the findings from this study added to the existing evidences on the benefits of CAOS regarding achieving improved clinical outcomes compared to conventional TKA. Additionally, 
compared to conventional TKA, the CAOS group demonstrated excellent short-term survivorship with zero case of early failure due to causes linked to postoperative mal-alignment.

This study may be limited by only presenting short-term outcomes, mid- to long- term performance of the CAOS TKA in the studied cohort remains to be shown. The short-term results reported by this study support the use of CAOS technology with the goals of better function and greater range of motion. Future steps of the study include longer follow-up periods and further recruitment of global study sites for a more robust patient cohort.

\begin{tabular}{lccc}
\hline \multicolumn{1}{c}{ CAOS } & Conventional & $\mathrm{P}$ \\
\hline $\mathrm{N} \quad 334$ & 461 & - \\
$\quad$ Enrolled & 215 & 350 & - \\
$\quad$ 1yr+ Follow-up Available & 20.7 & 38.1 & - \\
Mean Follow-up Period (months) & $67.5 \pm 9.2$ & $63.9 \pm 9.5$ & $<0.01$ \\
Age (year) & $30.7 \pm 7.1$ & $32.1 \pm 6.4$ & $<0.01$ \\
BMI (year) & $37.7 \%$ & $44.2 \%$ & 0.09 \\
Male (\%) & $89.2 \%$ & $85.8 \%$ & 0.22 \\
Primary OA (\%) & & & \\
\hline
\end{tabular}

B

\begin{tabular}{lccc}
\hline \multicolumn{1}{c}{ CAOS } & Conventional & $P$ \\
\hline Preop & & & \\
$\quad$ ROM & $111.2 \pm 15.8$ & $113.5 \pm 13.2$ & 0.07 \\
KSS Function & $49.2 \pm 20.4$ & $45.4 \pm 19.3$ & 0.02 \\
KSS Knee & $44.9 \pm 16.7$ & $45.2 \pm 16.3$ & 0.70 \\
KSS Pain & $8.9 \pm 11.9$ & $7.5 \pm 10.2$ & 0.11 \\
Postop & & & \\
ROM & $121.7 \pm 12.3$ & $117.5 \pm 12.2$ & $<0.01$ \\
KSS Function & $78.3 \pm 20.5$ & $71.8 \pm 23.3$ & $<0.01$ \\
KSS Knee & $80.5 \pm 20.1$ & $79.8 \pm 21.3$ & 0.63 \\
KSS Pain & $40.4 \pm 13.2$ & $42.1 \pm 12.6$ & 0.12 \\
\hline
\end{tabular}

Table 1. A) Details on demographics and characteristics of the study cohort. B) Summary of pre- and postoperative outcomes.
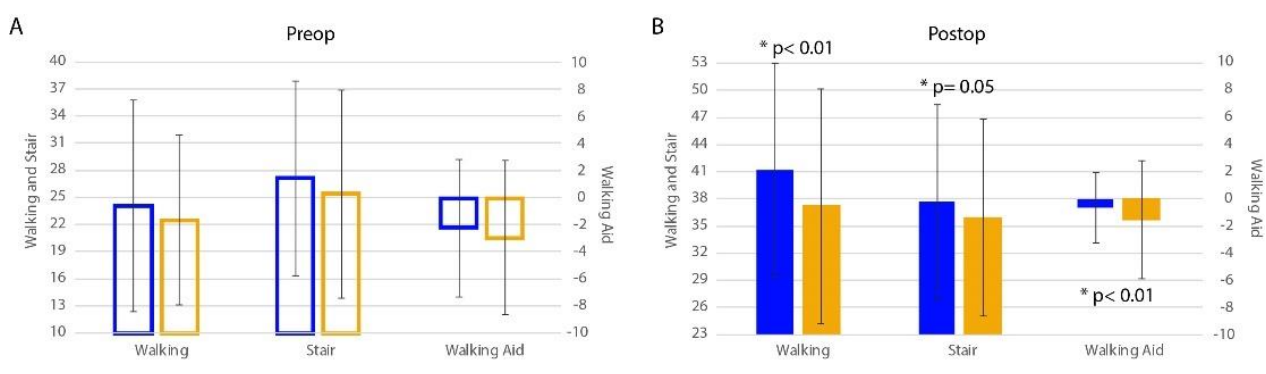

- CAOS Conventional

Table 2. Comparisons between CAOS and conventional TKA groups regarding sub-component measures under A) preop and B) postop KSS function. Preoperatively, all sub-component measures were statistically equivalent 
between CAOS and conventional groups. In contract, all sub-component measures demonstrated better outcome in the CAOS group compared to the conventional group. Charts on KSS knee was not shown due to no difference was found in the postoperative comparisons.

\section{References}

[1] Jenny JY, Clemens U, Kohler S, Kiefer H, Konermann W, Miehlke RK. Consistency of implantation of a total knee arthroplasty with a non-image-based navigation system: a case-control study of 235 cases compared with 235 conventionally implanted prostheses. J Arthroplasty. 2005;20(7): 832-9.

[2] Sparmann M, Wolke B, Czupalla H, Banzer D, Zink A. Positioning of total knee arthroplasty with and without navigation support: a prospective, randomised study. J Bone Joint Surg Br. 2003;85(6):830-5.

[3] Rebal BA, Babatunde OM, Lee JH, Geller JA, Patrick DA Jr, Macaulay W. Imageless computer navigation in total knee arthroplasty provides superior short term functional outcomes: a meta-analysis. J Arthroplasty. 2014;29(5):938-44.

[4] Johnson DR, Dennis DA, Kindsfater KA, Kim RH. Evaluation of total knee arthroplasty performed with and without computer navigation: a bilateral total knee arthroplasty study. J Arthroplasty. 2013;28(3):455-8.

[5] Hoffart HE, Langenstein E, Vasak N. A prospective study comparing the functional outcome of computer-assisted and conventional total knee replacement. J Bone Joint Surg Br. 2012

Feb;94(2):194-9. 